\title{
Treatment of Giant Intracranial Aneurysms: a Review Based on Experience from 286 Cases
}

\section{Tratamento dos aneurismas gigantes intracranianos: uma revisão baseada na experiência de 286 casos}

\author{
Atos Alves de Sousa ${ }^{1,2,3}$ José Lopes de Sousa Filho ${ }^{1}$ \\ ${ }^{1}$ Department of Neurosurgery of the Santa Casa de Belo Horizonte, \\ Belo Horizonte, MG, Brazil \\ 2 Faculdade de Ciências Médicas de Minas Gerais, Belo Horizonte, \\ MG, Brazil \\ ${ }^{3}$ Honorary President of the World Federation of Neurosurgical \\ Societies (WFNS), Rome, Italy
}

Arq Bras Neurocir 2015;34:295-303

\author{
Marcos Antônio Dellaretti Filho ${ }^{1,2}$
}

\begin{abstract}
Address for correspondence José Lopes de Sousa Filho, MD, Department of Neurosurgery of the Santa Casa de Belo Horizonte, Av. Francisco Sales, 1111, Sta Efigênia, Belo Horizonte, MG, Brazil CEP: 30150-221 (e-mail: joselopessousa@gmail.com).
\end{abstract}

\begin{abstract}
Keywords

- giant aneurysm

- complex aneurysm

- microneurosurgery

Resumo

Palavras-chave

- aneurisma gigante

- aneurisma complexo

- microcirurgia

Aneurysms are classified as giant when their largest diameter is equal to or greater than $25 \mathrm{~mm}$, which represents approximately $5-7 \%$ of intracranial aneurysms. Severe disease with poor natural history presents with $68 \%$ mortality in two years and $85 \%$ in five years for untreated cases. Thus, in the majority of cases, the patients require treatment, despite the risks of therapeutic options. We discuss the epidemiology, natural history, diagnosis, and treatment of giant aneurysms based on the experience of 286 operations and literature data.

Os Aneurismas são classificados como gigantes quando seu maior diâmetro é igual ou superior a $25 \mathrm{~mm}$ e representam aproximadamente de 5 a $7 \%$ dos aneurismas intracranianos. Trata-se de patologia grave com história natural ruim apresentando mortalidade de $68 \%$ em 2 anos e $85 \%$ em 5 anos para casos não tratados. Desta forma, na maioria dos casos, estes pacientes devem ser tratados apesar do alto risco das opções terapêuticas. Neste estudo, discutimos a epidemiologia, a história natural, o diagnóstico e o tratamento desta grave patologia baseado na experiência de 286 pacientes tratados e dados da literatura.
\end{abstract}

\section{Introduction}

Morley and Barr classify cerebral aneurysms as giant when their largest diameter is equal to or greater than $25 \mathrm{~mm}$. The arbitrary limit of $2.5 \mathrm{~cm}$ distinguishes an aneurysm group with clinical and pathological characteristics, natural history, and treatment, which differ from those of small and medium aneurysms. ${ }^{1-4}$

received

November 10, 2013

accepted

August 7, 2015

published online

October 22, 2015
The most common location is in the internal carotid artery (ICA), followed by the vertebrobasilar system (VBS), middle cerebral artery (MCA), and most recently, the anterior cerebral-anterior communicating artery (ACAACoA) complex. ${ }^{1,2,4,5}$

Manifestations include subarachnoid hemorrhage (SAH) and/or intracerebral hemorrhage (ICH) and, particularly, signs and symptoms related to mass effect on the adjacent

Copyright $(2015$ by Thieme Publicações License terms Ltda, Rio de Janeiro, Brazil

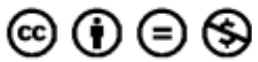


neural structures. Seizures and cerebral ischemia occur with less frequency. The natural history of giant cerebral aneurisms is very poor, since cases treated conservatively evolve to death in a few years. ${ }^{1,5-8}$

The treatment of such aneurysms is one of the greatest neurosurgical challenges. The difficulties involved are due to the size of the aneurysm, the presence of calcification and thrombus, the wide neck, and the incorporation of the artery from which they originate. Despite the increased difficulties in treatment and poor prognosis, they are potentially curable lesions and some type of therapeutic intervention should always be considered. ${ }^{9,10}$

\section{Epidemiology}

The literature reports that incidence of giant aneurysm ranges from 2 to $7 \%$ of all intracranial aneurysms. ${ }^{2,4,5}$ In the current series, incidence is $8.2 \%$, thus, 286 giant aneurysms out of a total of 3,500 patients operated between 1977 and 2013. Of these, 163 patients (57.0\%) were female and 123 (43.0\%) were male.

Giant aneurysms predominate in the female population at a frequency of 2:1 and affect all age groups, showing greater prevalence among 40 to 70 -year-olds. Several factors are associated with the development and rupture of these lesions, including being female, age, hypertension, smoking, and connective tissue diseases. ${ }^{1,2,4,11}$

\section{Natural History}

The natural history of giant aneurysms is very poor, since they are associated with high risk of bleeding, progressive neurological defects, and death, mainly a result of bleeding, but also of the mass effect and/or cerebral ischemia. The condition presents increased risk of SAH and higher mortality than small and medium aneurysms. Location in the posterior circulation is an isolated factor for poor prognosis. ${ }^{1,6-8,12,13}$

Drake $^{32}$ reported mortality of $68 \%$ at 2 years and $85 \%$ at 5 years for untreated giant aneurysms and the survivors showed severe neurological sequelae. According to the International Study of Unruptured Intracranial Aneurysms (ISUIA), ${ }^{11}$ the cumulative risk of bleeding is 40 to $50 \%$ at 5 years. Meningeal hemorrhage resulting from a giant aneurysm leads to higher mortality and serious neurological sequelae compared with smaller aneurysms (-Tables $\mathbf{1}$ and $\mathbf{2}$ ).

Table 1 Natural history of unruptured aneurysms ${ }^{11}$

\begin{tabular}{|l|l|}
\hline $\begin{array}{l}\text { Anterior circulation } \\
\text { aneurysms except ACoP }\end{array}$ & Annual risk of bleeding \\
\hline $7-12 \mathrm{~mm}$ & $0.5 \%$ \\
\hline $13-24 \mathrm{~mm}$ & $2.9 \%$ \\
\hline$\geq 25 \mathrm{~mm}$ & $8.0 \%$ \\
\hline
\end{tabular}

Table 2 Natural history of unruptured aneurysms ${ }^{11}$

\begin{tabular}{|l|l|}
\hline $\begin{array}{l}\text { Posterior circulation } \\
\text { aneurysms plus ACoP }\end{array}$ & Annual risk of bleeding \\
\hline $7-12 \mathrm{~mm}$ & $2.9 \%$ \\
\hline $13-24 \mathrm{~mm}$ & $3.7 \%$ \\
\hline$\geq 25 \mathrm{~mm}$ & $10.0 \%$ \\
\hline
\end{tabular}

The exception to poor prognosis are giant aneurysms of the extradural ICA (petrous and cavernous portion), which usually have a benign natural history. These can manifest as multiple cranial nerves paresis (III, IV, and VI), ischemia secondary to migration of intrasaccular thrombus, carotid cavernous fistula, and epistaxis. ${ }^{1,4,13}$

\section{Imaging Diagnosis}

Cranial computed tomography (CT) is the first examination that the clinician should request, as in all cases of cerebral aneurysms. This enables a diagnosis of SAH through the visualization of the actual size of the lesion and presence of thrombus and calcifications. The use of CT and/or magnetic resonance imaging (MRI) is essential for the determination of the thrombosed portion of the lesion, the exact size of the aneurysm, the presence of cerebral ischemia, and its relation with adjacent neural structures. ${ }^{10,15}$

Digital angiography with three-dimensional (3D) reconstruction remains the gold standard for the diagnosis and treatment planning of giant aneurysms. It allows for a dynamic study of the entire cerebral circulation as well as an evaluation of the location and portions of the affected vessel and the aneurysm neck for the balloon test occlusion (BTO). 3,10,16

An intraoperative angiography is very useful as a quality control method in giant aneurysm surgery. It allows the surgeon to correct the clips in the reconstruction of the aneurysm during surgery, avoiding stenosis at the artery origin or persistence of part of the aneurysm not excluded from circulation. All patients should undergo a study of cerebral circulation with postoperative digital angiography. Another intraoperative control option is video angiography with intravenous indocyanine using a microscope equipped with this technology. ${ }^{13,16,17}$

\section{Treatment}

The treatment should be performed in a neurosurgical center with experience in treating patients with cerebral aneurysms, which can perform both microsurgery and endovascular techniques. The treatment modalities are conservative, surgical, endovascular and combined. Treatment management of giant aneurysms should take into account the risks and benefits of each treatment option. ${ }^{7,11}$

There have not yet been any randomized clinical trials conducted on the topic. Current literature data refer to large series in select centers. The decision process for treating 
these lesions has been individualized; however, this should be discussed within a multidisciplinary team.

\section{Conservative Treatment (-Fig. 1)}

It is mainly indicated for asymptomatic patients with an extradural giant aneurysm of the intracavernous or petrous ICA. Patients with minor symptoms with a precarious health status or of advanced age may also undergo monitoring. Patients with symptoms of cerebral ischemia secondary to migration of an intrasaccular thrombus of a cavernous aneurysm that are not candidates for other treatments may be administered an antiplatelet agent. ${ }^{11,18,19}$

\section{Surgical Treatment}

Surgical treatment of giant aneurysms remains the optimal therapeutic choice in most cases. The aim is to exclude the aneurysm from circulation, maintaining the patency of the artery involved, eliminating the mass effects and conserving neurological functions. ${ }^{4,18,20}$ Factors that can complicate surgery include: a wide neck, afferent and efferent vessels encompassed by the aneurysm, the presence of intrasaccular thrombus, and calcifications in the aneurysm neck and the source artery. Paraclinoid aneurysms and those located in the posterior circulation represent a greater challenge. ${ }^{9,17,18}$
The patient's age and the morbidity of the treatment options factor into the decision over treatment. In contrast to poor natural history, several surgical series have reported good clinical outcomes in 58 to $84 \%$ of patients, with mortality rates ranging from 14 to $22 \%$. $^{13,17,18,20}$

In the current series of 286 giant aneurysms, we observe a good clinical outcome (Glasgow Outcome Scale; GOS 4 and 5) in $81.1 \%$ of cases and poor clinical outcome (GOS 1,2 , and 3 ) in $18.9 \%$. The results of the main surgical series (clipping, reconstruction and bypass) are presented in -Table 3.

Elderly patients with multiple comorbidities and aneurysms located in the posterior circulation tend to present a worse clinical outcome. The use of adjuvant therapies, such as temporary clipping, arresting the heart, deep hypothermia, and bypass, all present increased specific risks that must be considered. ${ }^{13,18,20,23}$

Lawton et $\mathrm{al}^{22}$ evaluated the results of surgical management in 141 intracranial giant aneurysms operated between 1997 and 2010, on patients with a mean age of 54 years old. The majority of patients presented symptoms of compression of the cranial nerves, while 23 patients presented cerebral hemorrhage. One hundred aneurysms were located in the anterior circulation, mainly in the ICA and the MCA, and 41 in the posterior circulation.

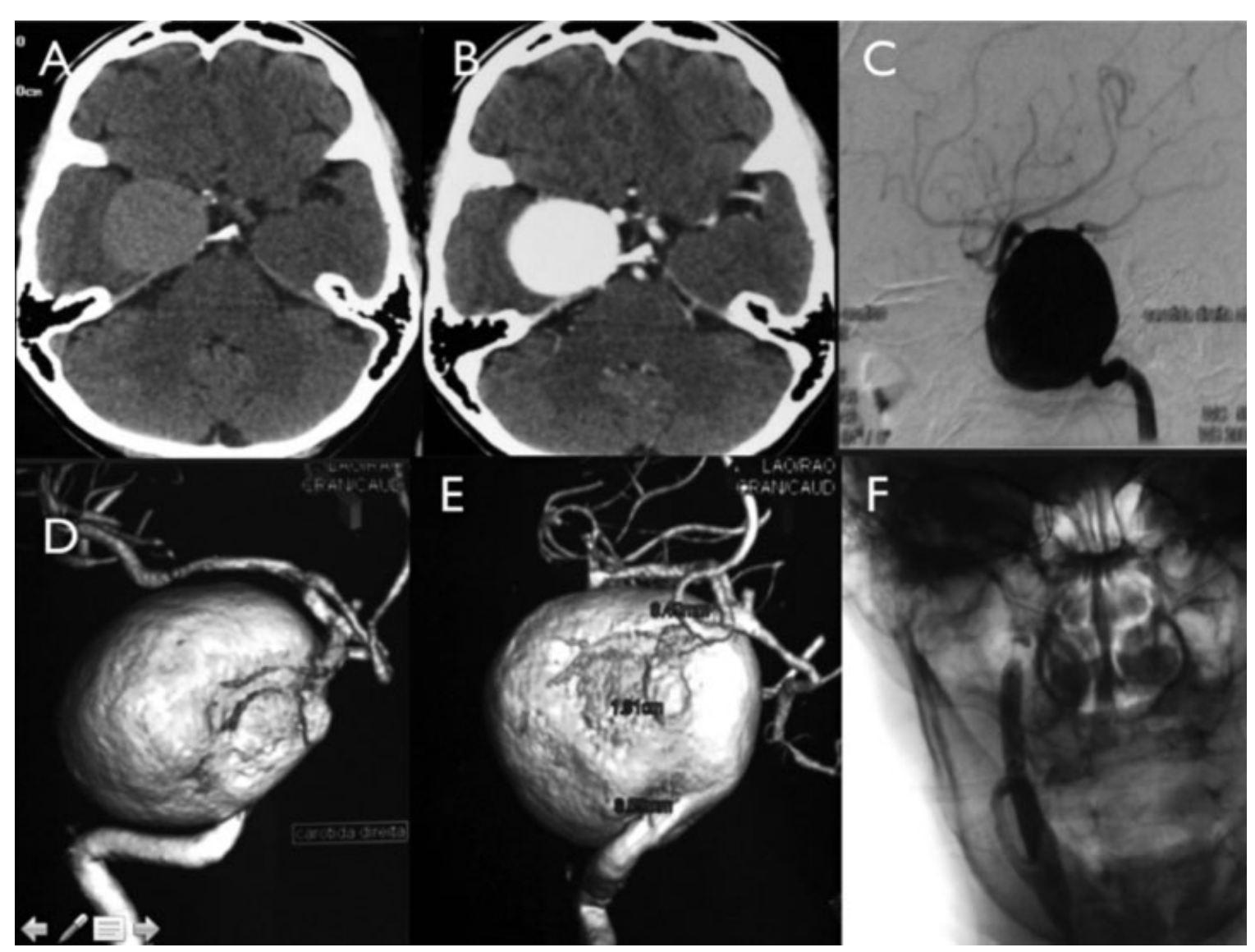

Fig. 1 Conservative treatment. Patient, 65 years old, presenting hypertension and diabetes with paresis of the III right cranial nerve. CT scan without $(A)$ and with (B) contrast show nodular lesion right temporal. Cerebral angiography in lateral (C) and 3D reconstruction (D and $E$ ) confirmed giant aneurysm of the cavernous internal carotid artery. Patient did not tolerate the balloon occlusion test (F). 
Table 3 Surgical morbidity and mortality of giant aneurysms in literature $4,17,18,21,22$

\begin{tabular}{|l|l|}
\hline Literature results & Percentage \\
\hline Good or excellent clinical outcome & $58-84 \%$ \\
\hline Mortality & $14-22 \%$ \\
\hline Rebleeding & $0-3 \%$ \\
\hline Retreatment & $0-1 \%$ \\
\hline
\end{tabular}

A direct approach with aneurysm neck clipping was the preferred primary treatment strategy, comprising 66 (46.8\%) of the cases. Alternatively, 72 (51.1\%) patients underwent an indirect approach. Bypass formed part of the treatment strategy in 54 (38.3\%) aneurysms, with the following distribution: highflow bypass in 14 patients (25.9\%); low-flow bypass in 11 patients (20.4\%); and 15 (27.8\%) intracranial bypasses in situ and other forms of bypass (reimplantation/reanastomosis) in 14 patients (25.9\%). During follow-up with control tests, 108 (76.6\%) aneurysms were completely occluded, 14 (9.9\%) had minimal residual aneurysm neck, and 16 (11.3\%) were incompletely occluded with aneurysm reversal or flow reduction. ${ }^{22}$

We observed good clinical outcomes (GOS 4 and 5) in 114 (80.9\%) patients. The mortality rate was 12.8 and the neurological morbidity rate related to treatment was $9.2 \%$. Factors identified for poor prognosis were location in the posterior circulation, clinical presentation with hemorrhage, and calcified aneurysms. ${ }^{22}$

\section{Proximal and Distal Ligation of the Feeding Artery (Hunterian Ligation and Surgical Trapping)}

Dandy first proposed trapping, which consists of ligation of the feeding artery proximally and distally to the aneurysm neck. This can be achieved using a surgical or endovascular approach, the latter involves using detachable balloons or coils. ${ }^{3,10,16}$

The BTO should precede the applicaiton of such techniques. As described by Matas ${ }^{10}$ in 1911 , temporary occlusion of the internal carotid artery provides valuable information for preoperative planning in cases of giant aneurysm. The parameters used are early arterial filling difference, simultaneous capillary venous time, and collateral circulation through the anterior communicating artery..$^{10,16,24}$ Induced hypotension is used to increase assay sensitivity, with reduction in blood pressure (BP) to $20 \%$ of the baseline value or $20 \mathrm{~mm} \mathrm{Hg}$ for 30 minutes, with serial neurological examination assessment. ${ }^{10,16,18,24}$

When the patient shows tolerance for BTO, this means that they present good cerebrovascular reserve and, therefore, should endure occlusion of the internal carotid artery with low morbimortality. Surgical or endovascular occlusion of the internal carotid can be performed without prior revascularization. ${ }^{10,18,24}$

According to Linskey et al, ${ }^{25}$ ligation of the internal carotid without prior collateral flow studies results in $25 \%$ morbidity and $12 \%$ mortality; whereas, when ligation is performed after collateral flow studies, morbidity is $4.7 \%$ and mortality is $0 \%$. Therefore, when the patient shows intolerance for BTO, a high-flow bypass is required prior to occlusion of the internal carotid to guarantee cerebral perfusion.

\section{Direct Clipping of the Aneurysm Neck or Reconstruction with Clips}

According to some authors, the ideal treatment for giant aneurysms remains the direct approach to the aneurysm using microsurgery. This is possible in $\sim 50-60 \%$ of cases. ${ }^{4,18,26}$ Occlusion of the aneurysm neck using clips has several peculiarities. The success of clipping a giant aneurysm depends on its morphology and anatomical complexity. The aneurysm must have a well-defined neck (- Figs. $\mathbf{2 , 3}$, and 4). When direct neck clipping is inviable, the use of reconstruction techniques is required to exclude the aneurysm from circulation (-Figs. 5 and $\mathbf{6}$ ). ${ }^{17,18,27}$

The presence of arterial branches incorporated into the aneurysm body, a wide neck, calcifications in the arterial wall, and/or aneurysm neck, all complicate the surgical treatment. Calcifications can expel clips or prevent their closure. Due to these characteristics, temporary clipping is used in most cases of giant aneurysm, allowing for dissection of the neural structures and manipulation of the bottom of the aneurysm, thus, also facilitating the application of clips. In giant partially thrombosed aneurysms, opening of the aneurysm sac to empty the clots (thrombectomy) is required for a definitive clipping of the neck. ${ }^{4,17,18}$

To minimize complications, neurosurgeons have relied on numerous "checking methods" during the intraoperative period to ensure aneurysm exclusion and prevent vessel stenosis. The most commonly used methods are intraoperative Doppler ultrasound and digital angiography. More recently, the advent of video angiography with intravenous indocyanine green has offered the neurosurgeon the same parameters albeit in a less invasive manner. ${ }^{12,17,22,28}$

Spagnuolo et $\mathrm{al}^{13}$ reviewed 145 cases of giant aneurysm treated with direct surgical approach to the aneurysm neck. Twenty-six patients presented cerebral hemorrhage. Patients with ruptured aneurysms presented $15 \%$ mortality and $10 \%$ morbidity; in contrast, patients with unruptured aneurysms presented $6.5 \%$ mortality and $15 \%$ morbidity. Complications mainly related to occlusion/ thrombosis of the affected vessel, with consequent cerebral ischemia, hemorrhagic phenomena, and clinical complications.

\section{Bypass with Proximal and Distal Occlusion}

Yasargil, ${ }^{11}$ in 1969, developed and introduced cerebral revascularization techniques into neurosurgery practice. A bypass can be one of two types, according to the resulting blood flow: high-flow or low-flow. The most commonly used type of revascularization in giant aneurysm surgeries is a highflow bypass between the cervical external carotid artery and one of the M2 branches of the middle cerebral artery, with the interposition of a radial artery or saphenous vein graft (-Fig. 7). It is indicated when the patient shows intolerance for BTO and no other options are viable. ${ }^{26,29}$ 


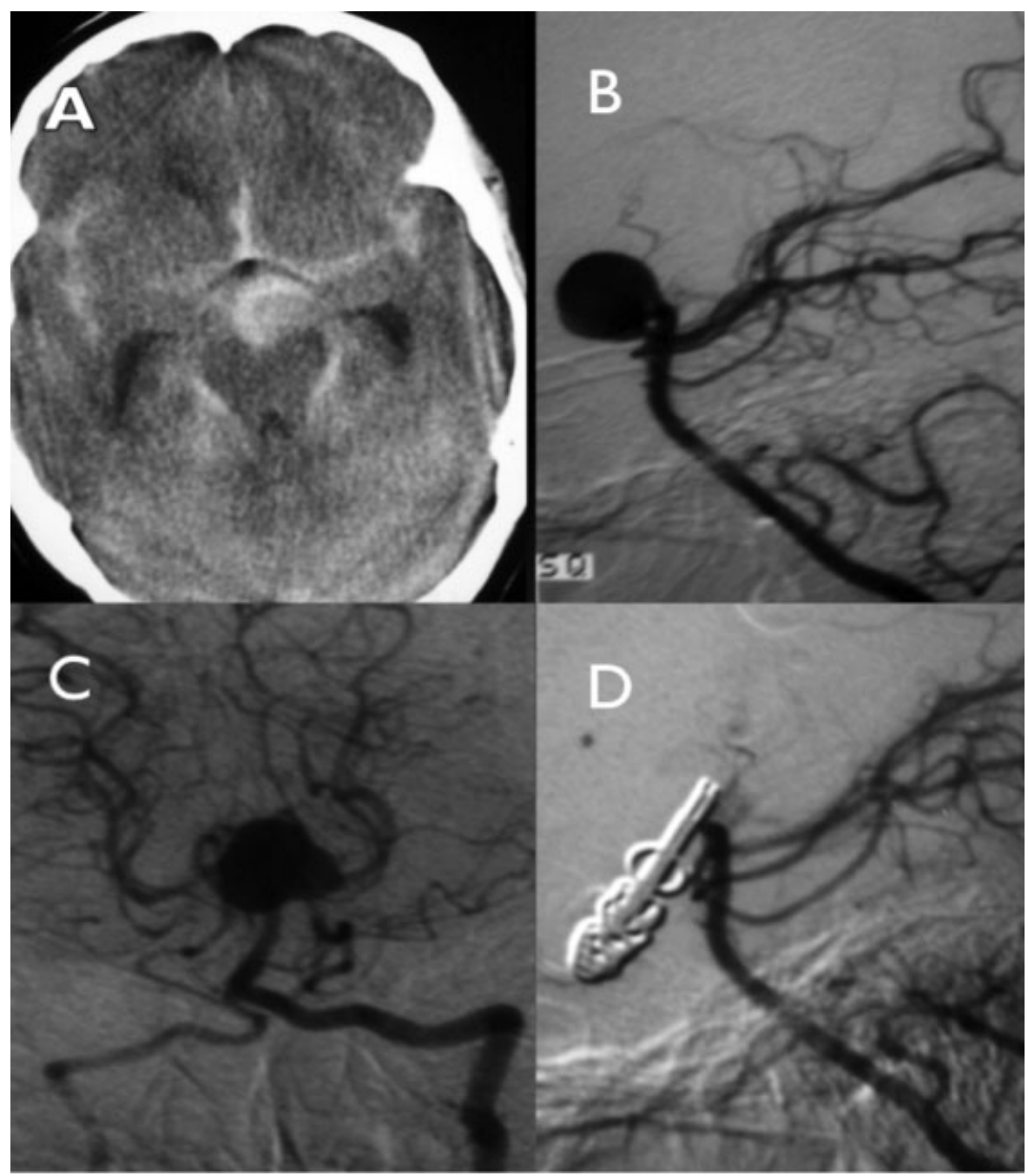

Fig. 2 Direct approach to neck of the brain aneurysm. Patient with subarachnoid hemorrhage diagnosed by CT scan without contrast (A). Cerebral angiography in lateral (B) and anteroposterior (C) shows a giant aneurysm at the apex of the basilar artery narrow neck. Performed microsurgical clipping with direct approach to the neck of the aneurysm through fronto-orbitozygomatic craniotomy. Cerebral angiography control (D) showing complete exclusion of the aneurysm with preservation of the posterior cerebral arteries.

Jafar et $\mathrm{al}^{21}$ analyzed the results of surgical treatment in 29 patients with giant aneurysms submitted to extracranialintracranial high-flow bypass with saphenous vein graft and immediate occlusion of the vessel with the aneurysm. Follow-up at 62 months showed 6\% neurological morbidity and $3 \%$ mortality with $100 \%$ occlusion of the aneurysm from circulation. The graft occlusion rate was $7 \%$ early and $0 \%$ late. The main complications related to ischemic phenomena, such as choroidal artery infarction and perforating arteries. The authors concluded that the use of extracranial-intracranial high-flow bypass with saphenous vein graft followed by occlusion of the affected vessel is a safe, effective treatment for giant aneurysms. ${ }^{21}$

\section{Endovascular Treatment}

The endovascular approach is a good treatment option for giant aneurysms, particularly for unruptured aneurysms, spindle aneurysms, located in the posterior circulation, and for elderly patients with multiple comorbidities. Despite the development of endovascular technologies in recent years, they still present certain limitations, including incomplete occlusion with high rates of recanalization, the need for retreatment, and occasional post-treatment bleeding. This approach does not solve pseudo-tumor compressive effects on the adjacent neural structures. ${ }^{19,27}$

Some factors intrinsic to giant aneurysms make them pathologies of difficult endovascular treatment, such as a wide neck, with incorporation of the feeding artery, intraluminal thrombosis, and the involvement of perforating.

Parkinson et $\mathrm{al}^{19}$ analyzed the clinical results of the largest endovascular series for treatment of giant aneurysms published since 1994, following the development of coils. They identified 316 patients submitted to endovascular treatment, in which $19 \%$ of cases manifested cerebral hemorrhage. The average of complete aneurysm occlusion was $57 \%$ of cases, with $7.7 \%$ mortality and $17.2 \%$ neurological morbidity during a mean clinical followup of 17.6 months. The recanalization rate was $27 \%$ in 238 aneurysms followed for 12.4 months. ${ }^{19}$ 


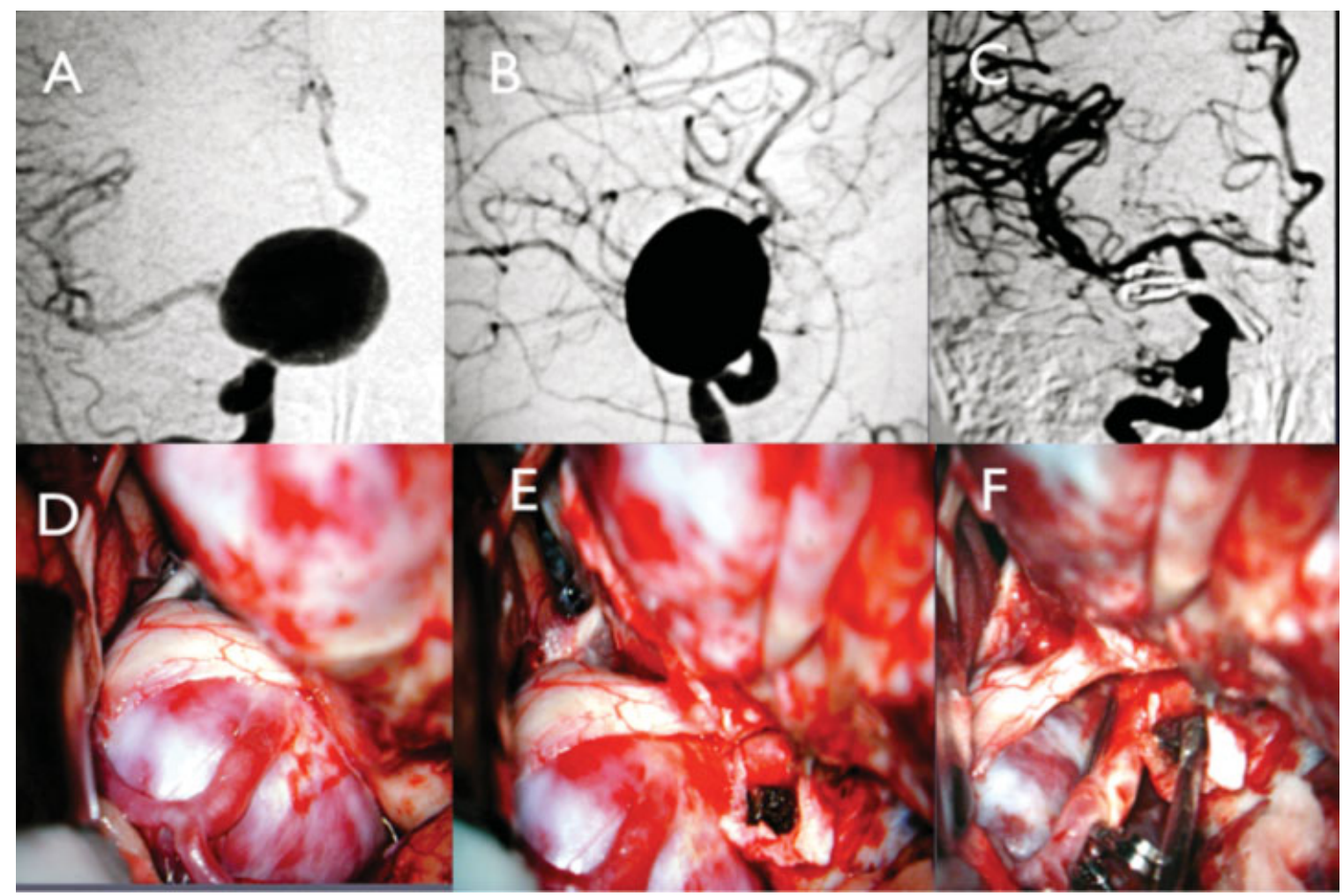

Fig. 3 Direct approach to neck of the brain aneurysm. Giant aneurysm intracranial of the internal carotid artery left evidenced by cerebral angiography AP (A) and lateral (B). Intraoperative findings revealed compression of optic pathways (D) and early proximal vascular control (E). Performed clipping of the aneurysmatic neck without vascular stenosis ( $F)$. Control angiography (C) with complete exclusion of the aneurysm from the circulation.

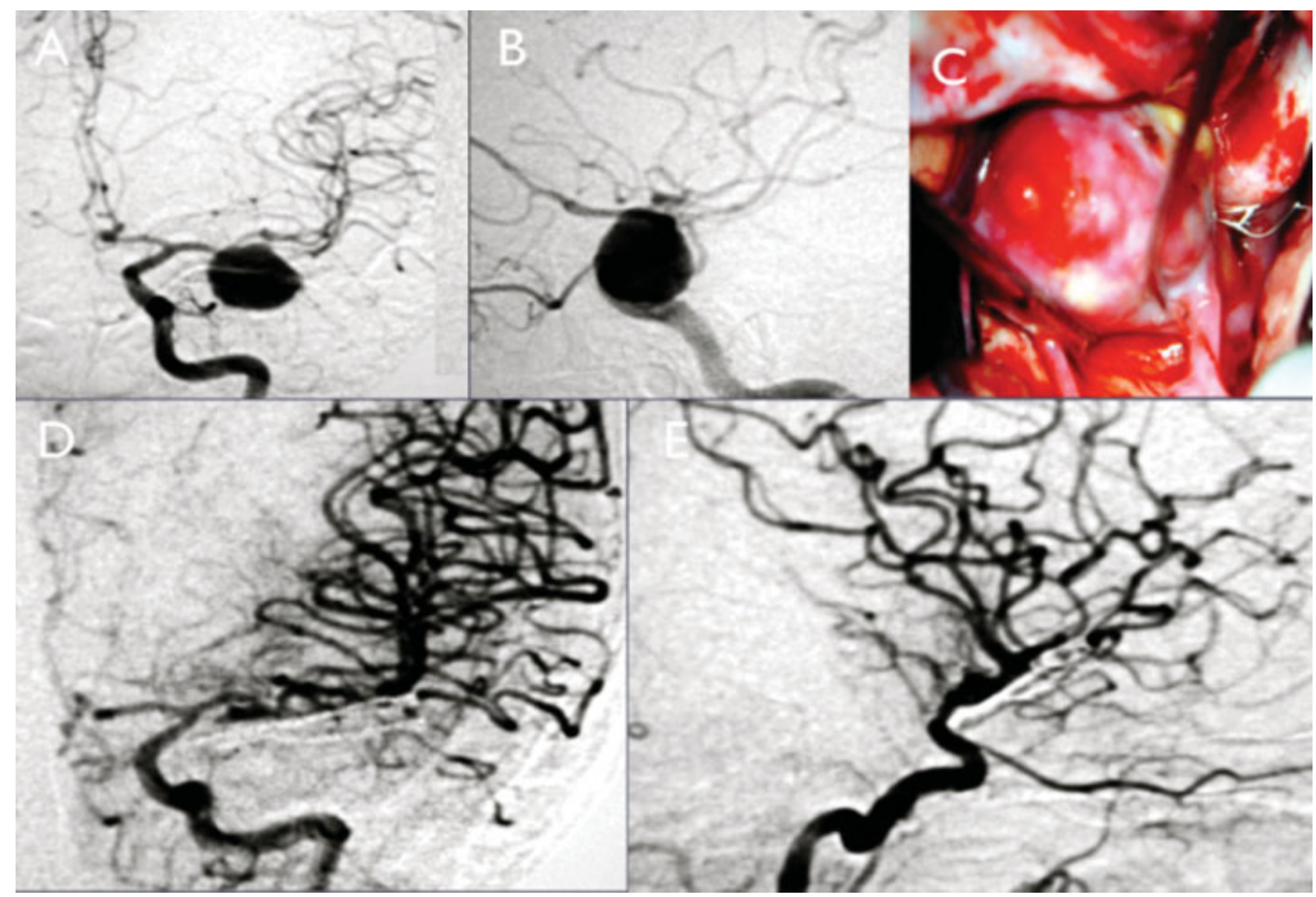

Fig. 4 Direct approach to neck brain aneurysm. Aneurysm of the middle cerebral artery (MCA) left with lower projection evidenced by cerebral angiography AP (A) and lateral (B). Intraoperative findings showing wide neck aneurysm of the MCA (C). Performed clipping of the aneurysm without stenosis confirmed by angiography control in AP (D) and lateral (E). 


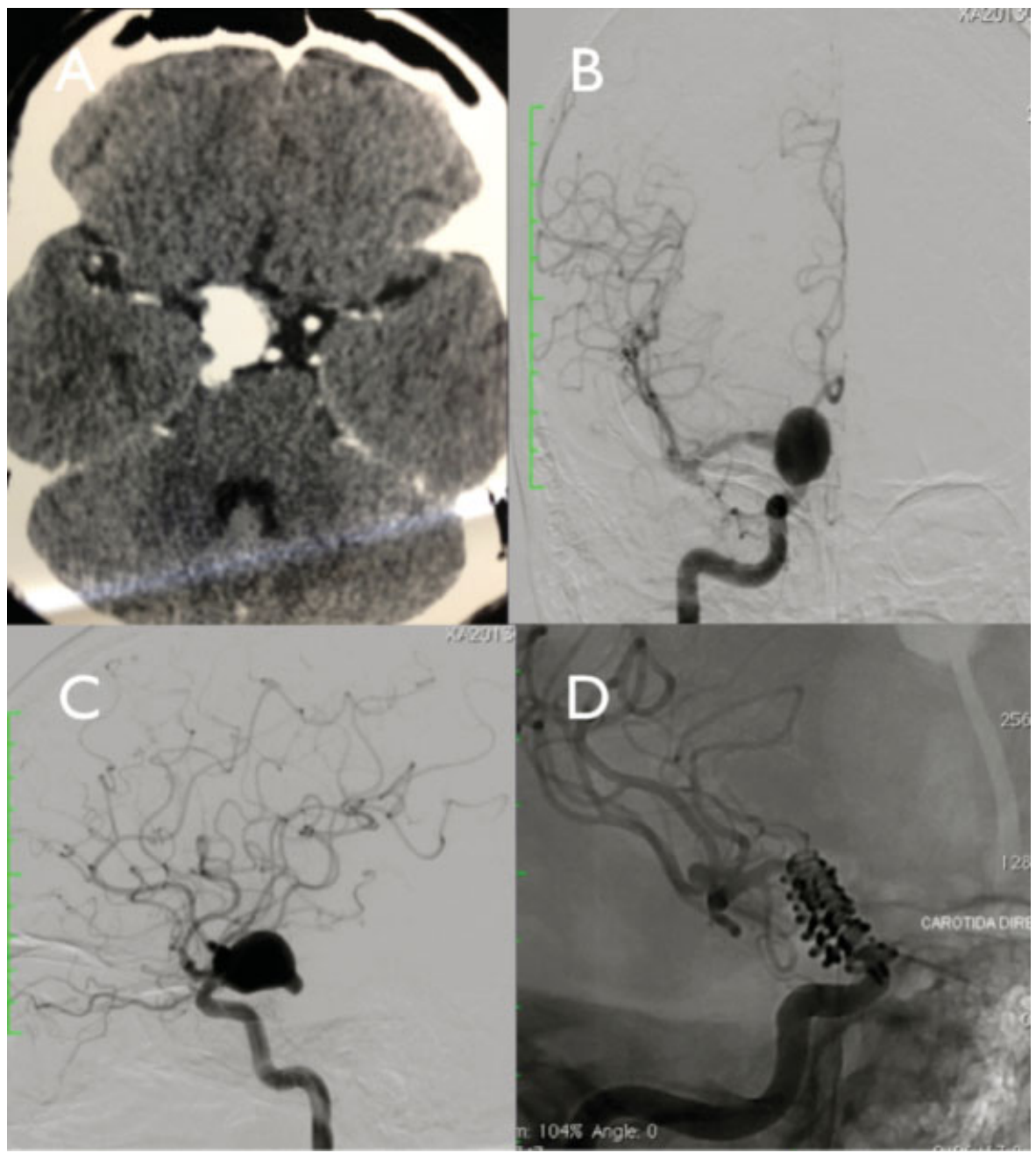

Fig. 5 Vascular reconstruction with multiple clips. CT scan (A) shows nodular lesion in the right parasellar region. Cerebral angiography in AP (B) and lateral $(C)$ show a giant aneurysm of the intracranial internal carotid artery. Performed vascular reconstruction with multiple fenestrated clips and control angiography (D) shows preservation of vascular flow.

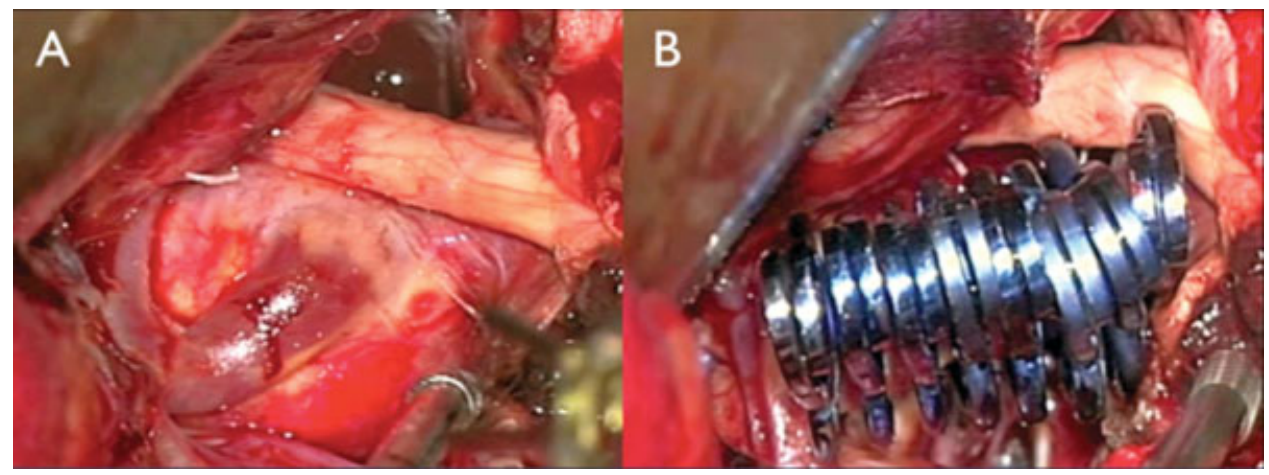

Fig. 6 Vascular reconstruction with multiple Clips. Intraoperative findings of vascular reconstruction with multiple fenestrated clips show compression of the optic pathways for aneurysm (A) and final aspect of the reconstruction of the internal carotid artery (B).

When analyzing the exclusive use of coils as a treatment strategy, only $43 \%$ of cases presented complete aneurysm occlusion, with $9 \%$ mortality and $24 \%$ major neurological morbidity. The recanalization rate was $55 \%$ in 164 giant aneurysms followed. The exclusive use of onyx, coil, and/or onyx stents showed similar results, making these viable strategies for the safe, definitive treatment of giant aneurysms. ${ }^{19}$

Endovascular occlusion of the affected vessel with coil following BTO proved to be safe and effective. In a review of 


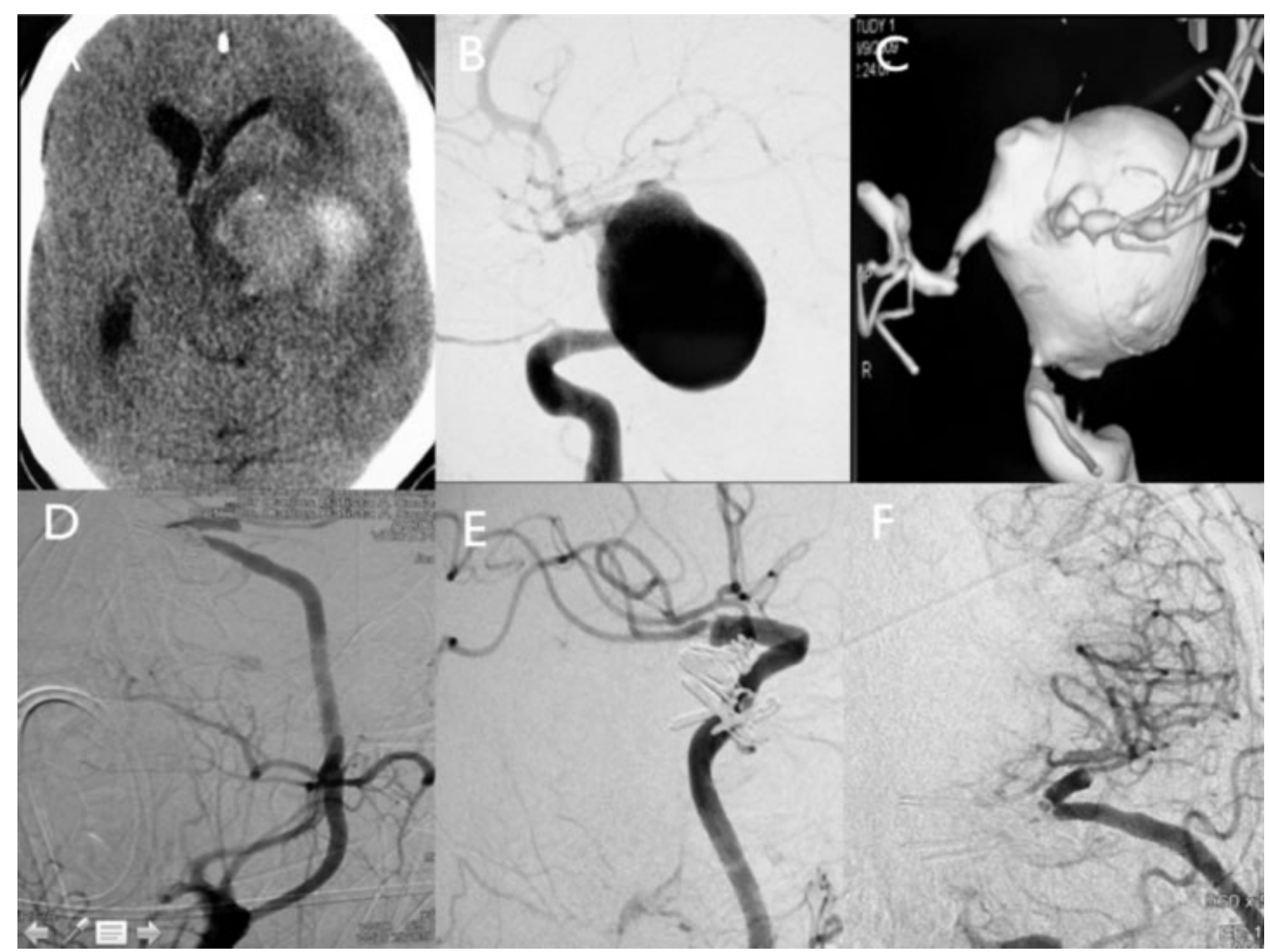

Fig. 7 Bypass and surgical trapping of the aneurysm. CT scan (A) with hemorrhage and mass effect. Cerebral angiography in AP (B) and threedimensional reconstruction (C) show the internal carotid artery giant aneurysm (ICA) left with involvement of afferent and efferent vessels. Performed bypass from the cervical external carotid artery to M2 branch of the middle cerebral artery (D and E). Control angiography showing excluded the aneurysm with good cerebral perfusion by shunt (F).

72 cases, Parkinson et al. verified $81 \%$ initial occlusion and $1 \%$ recanalization. ${ }^{19}$ Mortality rate was $7 \%$ of neurological morbidity followed for 14 months. ${ }^{19}$

Jahromi et $\mathrm{al}^{15}$ retrospectively analyzed the clinical and angiographic outcomes following endovascular treatment of 39 giant aneurysms. Ten patients presented ruptured aneurysms. Treatment with stents and coils was used in 25 aneurysms. An average of 1.9 sessions was required to treat each aneurysm, with 95\% angiographic occlusion of aneurysms and $75 \%$ patency of the affected vessel. The 30-day mortality was $8 \%$, with $20 \%$ permanent neurological morbidity.

Flow diverter stents are designed to induce redirection of blood flow near the aneurysm neck, preserving it in the affected vessel and its branches. They can be a good treatment option for basilar, petro-cavernous, and paraclinoid aneurysms, for which surgical options present particularly high morbimortality. Given the need for dual-antiplatelet therapy, the use of flow diverters is not recommended when the aneurysm is ruptured due to the higher incidence of hemorrhage and worse clinical outcome. ${ }^{23,30}$

The Pipeline for Uncoilable or Failed Aneurysms (PUFs) trial $^{20}$ evaluated the safety and efficacy of the pipeline embolization device (PED) in the treatment of large and giant aneurysms with a wide neck. The primary outcome was death or ipsilateral stroke within 180 days. The PED was used prospectively in 108 patients in 10 centers on 22 (20.4\%) giant aneurysms and 85 (78.7\%) large aneurysms. The complete occlusion rate was $73.6 \%$ at 180 days and $86.8 \%$ at one year. The primary outcome for six (5.6\%) patients was death or ischemic stroke, while 44 patients presented serious adverse effects, including cerebral hemorrhage (4.7\%), amaurosis fugax (4.7\%), headache (4.7\%), non-neurological bleeding (4\%), ischemic stroke (3.7\%), carotid cavernous fistula (1.9\%), cardiac arrhythmia (2.8\%), and carotid occlusion $(0.9 \%){ }^{20}$

\section{Prognostic Factors}

Darsaut et $\mathrm{al}^{31}$ analyzed the clinical and angiographic predictors of clinical and neurological outcomes following the treatment of very large $(20-24 \mathrm{~mm})$ or giant cerebral aneurysms ( $>25 \mathrm{~mm}$ ). They analyzed 184 aneurysms, 99 of which were giant, submitted to treatment and follow-up at Stanford University.

After multivariate analysis, the main prognostic factors for poor clinical outcome were a modified Rankim Scale score greater than 2, aneurysms larger than $25 \mathrm{~mm}$, and 
aneurysms located in the posterior circulation. Risk factors for incomplete angiographic occlusion were fusiform morphology, aneurysms located in the posterior circulation, and aneurysms submitted to endovascular treatment. Patients who presented incomplete occlusion in the angiographic follow-up showed high levels of SAH and increased mortality during follow-up, compared with completely occluded aneurysms. ${ }^{31}$

\section{Conclusions}

Despite important advances in diagnostic imaging methods and the development of microsurgical and endovascular techniques, the treatment of giant aneurysms remains a challenge for neurosurgery. Treatment should be individualized and discussed among a multidisciplinary team. The advent of new endovascular technologies, such as flow-diverting stents is a promising advance in the treatment of this pathology, with good short and medium term results. The surgical treatment of these lesions remains the principal therapeutic modality in selected centers, due to the durability, costs, low rates of recanalization, and good clinical outcomes obtained.

\section{References}

1 Barrow DL, Alleyne C. Natural history of giant intracranial aneurysms and indications for intervention. Clin Neurosurg 1995;42:214-244

2 Battaglia R, Pasqualin A, Da Pian R. Italian cooperative study on giant intracranial aneurysms: 1 . Study design and clinical data. Acta Neurochir Suppl (Wien) 1988;42:49-52

3 Morley TP, Barr HW. Giant intracranial aneurysms: diagnosis, course, and management. Clin Neurosurg 1969;16:73-94

4 Peerless SJ, Wallace MC, Drake CG. Giant intracranial aneurysms. In: Youmans JR (Ed). Neurological Surgery. 3rd ed. Philadelphia, PA: WB Saunders; 1900;3:1742-1763

5 Rosta L, Battaglia R, Pasqualin A, Beltramello A. Italian cooperative study on giant intracranial aneurysms: 2. Radiological data. Acta Neurochir Suppl (Wien) 1988;42:53-59

6 Christiano LD, Gupta G, Prestigiacomo CJ, Gandhi CD. Giant serpentine aneurysms. Neurosurg Focus 2009;26(5):E5

7 Molyneux AJ, Kerr RS, Yu LM, et al; International Subarachnoid Aneurysm Trial (ISAT) Collaborative Group. International subarachnoid aneurysm trial (ISAT) of neurosurgical clipping versus endovascular coiling in 2143 patients with ruptured intracranial aneurysms: a randomised comparison of effects on survival, dependency, seizures, rebleeding, subgroups, and aneurysm occlusion. Lancet 2005;366(9488):809-817

8 Piepgras DG, Khurana VG, Whisnant JP. Ruptured giant intracranial aneurysms. Part II. A retrospective analysis of timing and outcome of surgical treatment. J Neurosurg 1998;88(3):430-435

9 Lawton MT, Spetzler RF. Surgical strategies for giant intracranial aneurysms. Neurosurg Clin N Am 1998;9(4):725-742

10 Matas R. I. Testing the Efficiency of the Collateral Circulation as a Preliminary to the Occlusion of the Great Surgical Arteries. Ann Surg 1911;53(1):1-43

11 Wiebers DO, Whisnant JP, Huston J III, et al; International Study of Unruptured Intracranial Aneurysms Investigators. Unruptured intracranial aneurysms: natural history, clinical outcome, and risks of surgical and endovascular treatment. Lancet 2003;362(9378):103-110

12 Ferguson GG. Physical factors in the initiation, growth, and rupture of human intracranial saccular aneurysms. J Neurosurg 1972;37(6):666-677
13 Jaume A, Salle F, Fernandez M, Cobrera V, Aramburu I, Spagnuolo E. Surgical Treatment of Giant Intracranial Aneurysms: Series of 145 cases. J Bras Neurocirurg. 2013;24(3):212-219

14 Drake CG, Peerless SJ. Giant fusiform intracranial aneurysms: review of 120 patients treated surgically from 1965 to 1992 . J Neurosurg 1997;87(2):141-162

15 Jahromi BS, Mocco J, Bang JA, et al. Clinical and angiographic outcome after endovascular management of giant intracranial aneurysms. Neurosurgery 2008;63(4):662-674, discussion 674675

16 Lawton MT, Sanai N. Microsurgical management of giant intracranial aneurysms. In: Youmans JR (Ed). Neurological Surgery. 6th ed. Philadelphia, PA: WB Saunders; 2011:3953-3971

17 Lawton MT, Spetzler RF. Surgical management of giant intracranial aneurysms: experience with 171 patients. Clin Neurosurg 1995;42:245-266

18 Hanel RA, Spetzler RF. Surgical treatment of complex intracranial aneurysms. Neurosurgery 2008;62(6, Suppl 3):1289-1297, discussion 1297-1299

19 Parkinson RJ, Eddleman CS, Batjer HH, Bendok BR. Giant intracranial aneurysms: endovascular challenges. Neurosurgery 2006; 59(5, Suppl 3):S103-S112, discussion S3-S13

20 Becske T, Kallmes DF, Saatci I, et al. Pipeline for uncoilable or failed aneurysms: results from a multicenter clinical trial. Radiology 2013;267(3):858-868

21 Jafar JJ, Russell SM, Woo HH. Treatment of giant intracranial aneurysms with saphenous vein extracranial-to-intracranial bypass grafting: indications, operative technique, and results in 29 patients. Neurosurgery 2002;51(1):138-144, discussion 144-146

22 Sughrue ME, Saloner D, Rayz VL, Lawton MT. Giant intracranial aneurysms: evolution of management in a contemporary surgical series. Neurosurgery 2011;69(6):1261-1270, discussion 12701271

23 Leung GKK, Tsang ACO, Lui WM. Pipeline embolization device for intracranial aneurysm: a systematic review. Clin Neuroradiol 2012;22(4):295-303

24 Standard SC, Ahuja A, Guterman LR, et al. Balloon test occlusion of the internal carotid artery with hypotensive challenge. AJNR Am J Neuroradiol 1995;16(7):1453-1458

25 Linskey ME, Jungreis CA, Yonas $\mathrm{H}$, et al. Stroke risk after abrupt internal carotid artery sacrifice: accuracy of preoperative assessment with balloon test occlusion and stable xenon-enhanced CT. AJNR Am J Neuroradiol 1994;15(5):829-843

26 Yasargil MG. Giant intracranial aneurysms. Microneurosurgery II: Clinical considerations, surgery of the intracranial aneurysms and results. New York: Thieme-Stratton; 1984:296-304

27 Ponce FA, Albuquerque FC, McDougall CG, Han PP, Zabramski JM, Spetzler RF. Combined endovascular and microsurgical management of giant and complex unruptured aneurysms. Neurosurg Focus 2004;17(5):E11

28 Cantore G, Santoro A, Guidetti G, Delfinis CP, Colonnese C, Passacantilli E. Surgical treatment of giant intracranial aneurysms: current viewpoint. Neurosurgery 2008;63(4, Suppl 2): 279-289, discussion 289-290

29 Peerless SJ, Ferguson GG, Drake CG. Extracranial-intracranial (EC) IC) bypass in the treatment of giant intracranial aneurysms. Neurosurg Rev 1982;5(3):77-81

30 Nelson PK, Lylyk P, Szikora I, Wetzel SG, Wanke I, Fiorella D. The pipeline embolization device for the intracranial treatment of aneurysms trial. AJNR Am J Neuroradiol 2011;32(1): $34-40$

31 Darsaut TE, Darsaut NM, Chang SD, et al. Predictors of clinical and angiographic outcome after surgical or endovascular therapy of very large and giant intracranial aneurysms. Neurosurgery 2011; 68(4):903-915, discussion 915

32 Drake CG. Giant intracranial aneurysms: experience with surgical treatment in 174 patients. Clin Neurosurg 1979;26:12-95 